$42^{\text {nd }}$ AIAA Aerospace Sciences Meeting and Exhibit

Reno, Nevada

5-8 January 2004

\title{
DEVELOPMENT OF A TWO-WAY COUPLED MODEL FOR TWO PHASE RAREFIED FLOWS
}

\author{
Jonathan M. Burt* and Iain D. Boyd ${ }^{\dagger}$ \\ Department of Aerospace Engineering \\ University of Michigan, Ann Arbor, MI 48109
}

\begin{abstract}
Based on a previously published model for momentum and energy transfer to a spherical solid particle from a locally free molecular gas, a procedure is outlined for the simulation of one-way coupled two phase flows involving a nonequilibrium gas and a dilute solid particle phase. Following a simple analysis of interphase collision dynamics, the procedure is extended for use with a range of nonspherical particles. An extensive modification to this method is proposed to allow the modeling of two-way coupled flows, and a representative test case is used to verify that momentum and energy are conserved. The method described here is thought to be the first to allow for the simulation of two-way coupled two phase rarefied flows, and holds promise as a tool in the analysis of a variety of high altitude plume flows.
\end{abstract}

\section{INTRODUCTION}

Over the past several decades, much research has been focused on the distribution and properties of solid particles in rocket nozzle, spacecraft thruster, and spacecraft fuel venting flows ${ }^{1}$. A variety of particle types can be found in such flows, including soot, particles of ice or frozen fuel condensates, molecular clusters, and alumina. This last type has been the subject of several recent studies ${ }^{2-6}$, and is extremely important in the analysis of solid propellant rocket plumes. Alumina particles usually account for a large mass fraction among the constituents ejected through a solid rocket nozzle, and are often the dominant contributor to the plume radiation signature. Analysis and prediction of the optical properties of the plume are therefore highly dependent on the accuracy of algorithms for consideration of the particle phase. Furthermore, alumina particles have been shown to develop significant velocity and temperature lags within both the nozzle and plume, and may influence the overall performance and efficiency of the rocket motor. Particle impingement on nozzle walls or other surfaces may also be important considerations, and can affect nozzle efficiency or system reliability. In addition, the particles may significantly influence the properties of

${ }^{*}$ Graduate student, AIAA student member.

${ }^{\dagger}$ Professor, AIAA associate fellow. the surrounding gas, so that flow characteristics are governed by complex two-way coupling between the two phases.

These same effects may also be found in other multiphase plume and free expansion flows. In liquidpropellant rocket plume flows, soot particles often contribute significantly to the radiation signature and may reduce engine performance. Particles found in spacecraft thruster or fuel venting flows can impinge on and damage exposed surfaces. For these reasons, there is a desire for accurate methods to model the solid particle phase in solid propellant rocket plumes, and in other rocket exhaust, spacecraft thruster, and fuel venting flows.

Existing procedures for simulating such flows use Computational Fluid Dynamics (CFD) techniques to model the gas phase. The most ambitious studies currently in the literature consider the gas using three dimensional or axisymmetric finite volume shockcapturing methods ${ }^{7,8}$, which allow for the accurate simulation of certain nonequilibrium phenomena expected in rocket exhaust plumes at low altitudes. However, none of these simulation methods are valid for high altitude two phase plumes, where the gas may exhibit highly nonequilibrium behavior through much of the flowfield, and where virtually all CFD-based methods will develop significant inaccuracy. For the special case of two phase free expansion flow into a vacuum, these methods are characterized by numerical divergence, and the determination of any solution may be impossible.

An alternate starting point for high altitude plume simulations is the Direct Simulation Monte Carlo (DSMC) method $^{9}$, which models the gas phase as a large collection of computational particles and makes no assumptions of continuum or quasi-equilibrium gas flow. This method has in the past been used extensively to simulate plumes from high altitude rockets or spacecraft thrusters ${ }^{10,11}$, and has been shown to allow for a high degree of accuracy in the characterization of gas properties in such flows. In a recent paper by Gallis et al. ${ }^{12}$, an extension of the DSMC method is proposed to enable the simulation of rarefied flows involving a dilute and chemically inert solid particle phase. This method has been fully implemented within the existing

American Institute of Aeronautics and Astronautics

Approved for public release; distribution unlimited 
DSMC code MONACO ${ }^{13}$ and modified to allow for flows involving a diatomic gas. The implementation and validation of this method are discussed in a previous paper $^{14}$, where comparisons are made with results from an experimental study on the aerodynamic focusing of a particle beam ${ }^{15}$.

One major assumption of the Gallis method is that only one-way coupling calculations are required, so that the particle phase will have a negligible influence on the gas. As discussed above, this assumption is often invalid for the solid propellant rocket plume flows, where interphase momentum and energy transfer may significantly alter the gas properties through much of the simulation domain. These effects are incorporated into standard CFD codes for low-altitude analysis of solid-propellant rocket plumes, but it is thought that no approach has ever been used to model two-way coupled plumes at high altitudes, where the highly nonequilibrium nature of the gas prevents accurate simulation using a CFD-based approach. A modification to the Gallis method, which allows twoway coupling between the gas and particle phases, can potentially overcome this inherent limitation of CFD, and extend the altitudes and flow regimes for which two phase plume flows may be accurately modeled.

This paper presents a general set of procedures through which a solid particle phase may be included within a DSMC simulation, in order to model two phase rarefied flows. First, the one-way coupling method of Gallis et al. is discussed, and a summary is provided for the implementation of this method as described in Ref. (14). Particle shape effects are then considered, and the method is extended to allow for simulations involving a range of nonspherical particles. Following a detailed analysis of gas molecule behavior during an interphase collision, a procedure is outlined to enable two-way coupling between the particles and gas. This new method is then applied to model a test case, for which conditions are similar to those expected in a small solid propellant rocket flow. Simulation results are discussed, and it is shown that the method presented here is consistent with the method of Gallis et al.

\section{ONE-WAY COUPLING MODEL}

As described in Ref. (14), the Gallis model is used within a DSMC simulation to calculate the rates of momentum and energy transfer from a locally free molecular gas to a spherical solid particle. Every computational gas molecule assigned to the same grid cell as the solid particle is modeled as a large homogeneous collection of actual gas molecules, a fraction of which will collide with the particle during each time step. As implemented in Ref. (14), the gas molecules which do collide are then either reflected specularly off the particle surface, or are diffusely reflected with full thermal accommodation to the particle temperature.

Among the basic assumptions of the model are that the particle temperature is spatially uniform (i.e. the particle Biot number is assumed to be much less than one) and that the solid particle phase is dilute, so collisions between solid particles are infrequent and can be neglected. The contribution to the interphase energy transfer rate of collision-induced changes in the particle kinetic energy is assumed to be negligible, so that the rate of change in particle thermal energy will equal the total rate at which energy is transferred to the particle from the gas. Through considerations of momentum and energy conservation, it can be shown that this assumption is valid if the particle is much more massive than molecules in the surrounding gas, as is the case in all flows of interest here. It is also assumed that collisions between reflected gas molecules and incident molecules will have a negligible influence on interphase collision properties, so that the surrounding flow can be modeled as locally free molecular for calculations of momentum and energy transfer to the particle. The assumption of locally free molecular flow generally requires that the particle Knudsen number, defined as the ratio of the local gas mean free path to the effective particle radius (described below), be of order one or greater. These assumptions are found to hold over a wide range of flow regimes, and are generally valid for the two phase free expansion flows of interest.

It is also assumed that the particle is a perfect sphere. This assumption can be relaxed, to include a range of nonspherical particles found under a wide variety of flow conditions. The consideration of nonspherical particles is discussed in detail below. One last assumption of the Gallis method is that the particle phase has a negligible effect on the surrounding gas. This assumption can be relaxed as well; while the Gallis method addresses only the transfer of momentum and energy to the particle resulting from interphase collisions, a more general model described below also considers the influence of these collisions on the gas.

As discussed in Ref. (14), the algorithm for including solid particles in a DSMC simulation is based on a decoupling of interphase momentum and energy transfer from the temporal variation in particle properties. The total rates of momentum and energy transfer to a particle are calculated during each time step, and afterward the temperature, velocity, and position of the particle are modified. Note that vibrational excitation of a polyatomic gas is unlikely to have a significant influence on interphase energy transfer for all flow conditions of interest, so the vibrational terms in the energy transfer equation in Ref. (14) can be neglected. With this modification, the following equations are used to compute the rates of 
energy and momentum addition to a solid particle due to the presence of a single DSMC computational gas molecule within the same grid cell:

$$
\begin{aligned}
& \mathbf{F}_{\mathrm{p}}=\frac{\pi \mathrm{R}_{\mathrm{p}}^{2} \mathrm{~N}_{\mathrm{g}}}{\mathrm{V}_{\mathrm{c}}}\left(\mathrm{mc}_{\mathrm{r}}+\frac{\tau}{3} \sqrt{2 \pi \mathrm{m} \mathrm{k}_{\mathrm{B}} \mathrm{T}_{\mathrm{p}}}\right) \mathbf{u}_{\mathrm{r}} \\
& \dot{\mathrm{Q}}_{\mathrm{p}}=\frac{\pi \mathrm{R}_{\mathrm{p}}^{2} \tau \mathrm{N}_{\mathrm{g}} \mathrm{c}_{\mathrm{r}}}{\mathrm{V}_{\mathrm{c}}}\left(\frac{1}{2} \mathrm{mc}_{\mathrm{r}}^{2}+\mathrm{e}_{\mathrm{rot}}-\left(2+\frac{1}{2} \Lambda\right) \mathrm{k}_{\mathrm{B}} \mathrm{T}_{\mathrm{p}}\right)
\end{aligned}
$$

Here $R_{p}$ is the radius of a spherical particle (or the effective radius, as defined below, of a nonspherical particle), $\mathrm{N}_{\mathrm{g}}$ is the number of actual gas molecules represented by the computational molecule, $\tau$ is the thermal accommodation coefficient for the particle surface, $V_{c}$ is the cell volume, $m$ is the mass of a single gas molecule, $\mathbf{u}_{\mathrm{r}}$ is the relative velocity of the gas molecule with respect to the particle, $\mathrm{c}_{\mathrm{r}}$ is the absolute value of $\mathbf{u}_{\mathrm{r}}, \mathrm{k}_{\mathrm{B}}$ is Boltzmann's constant, $\mathrm{T}_{\mathrm{p}}$ is the particle temperature, $\Lambda$ is the number of rotational degrees of freedom of the gas, and $e_{\text {rot }}$ is the rotational energy for a single gas molecule. Note that, for simplicity, the word "molecule" is used here to describe either a polyatomic gas molecule or a single atom in a monatomic gas.

The total force and heat transfer rate on a solid particle are found during each time step by evaluating Eqs. (1) and (2) for all computational gas molecules in the cell, and summing the resulting values. The particle velocity is then altered by the product of the total force vector and a factor $\Delta \mathrm{t} / \mathrm{M}_{\mathrm{p}}$, where $\Delta \mathrm{t}$ is the time step size and $\mathrm{M}_{\mathrm{p}}$ is the particle mass. Similarly, the particle temperature is altered by the product of the total heat transfer rate and $\Delta t /\left(c_{p} M_{p}\right)$ where $c_{p}$ is the particle specific heat. Once new values of the particle velocity $\mathbf{u}_{\mathrm{p}}$ and temperature $T_{\mathrm{p}}$ have been determined, the particle is moved through the grid by a distance $\mathbf{u}_{\mathrm{p}} \Delta \mathrm{t}$. If necessary, further calculations are then performed to reassign the particle to a new cell, account for a collision with a solid wall, or remove the particle from the simulation. As implemented for multiphase flow simulations, numerous particles are tracked simultaneously through the grid, each representing a large number $\mathrm{N}_{\mathrm{p}}$ of actual solid particles. Cell-averaged particle properties, such as number density, temperature, and mean velocity, are averaged over several thousand time steps to determine the overall characteristics of the particle phase.

\section{CONSIDERATION OF NONSPHERICAL PARTICLES}

While experimental studies have shown that alumina particles in solid rocket exhaust flows tend to be nearly spherical, nonspherical particles are prominent in other flows of interest, including liquid propellant rocket plume flows and spacecraft fuel venting flows ${ }^{1,16}$. Subject to the above assumptions on which the method of Gallis et al. is based, these nonspherical particles can also be considered through the following analysis. First, a few additional assumptions must be made for any nonspherical particle: The particle is assumed to have a convex shape, so that no outward vector originating at a point on the particle surface will intersect the particle surface at any other point. While this will not be true for very complex particles such as soot agglomerates, it is generally valid for a wide range of particles, including many particles formed during spacecraft fuel venting. In addition, the particle is assumed to move through the gas with an isotropic distribution of orientations relative to any fixed coordinate system, so that no one orientation is more likely than any other. While this implies that a nonspherical particle must be rotating, it is further assumed that any rotation effects - particle angular momentum, the side force due to an asymmetric surface pressure distribution, rotationinduced time variation in interphase momentum and heat transfer, etc. - are relatively small and can be neglected. These assumptions are expected to be valid over all relevant flow regimes for a variety of particle types.

Subject to the above assumptions, the convective heat transfer rate between a solid particle and the surrounding gas will depend on the particle shape only through the value of the average collision cross section for interphase collisions, given here as $\sigma$. Furthermore, the average momentum transfer rate will depend on the particle shape only through the value of $\sigma$ and through the distribution function of the collision angle $\theta$. Here $\theta$ is defined as the angle between the relative velocity $\mathbf{u}_{\mathrm{r}}=\mathbf{u}_{\mathrm{m}}-\mathbf{u}_{\mathrm{p}}$ of an incident gas molecule and an outward normal vector at the collision point on the particle surface, where $\mathbf{u}_{\mathrm{m}}$ and $\mathbf{u}_{\mathrm{p}}$ are the velocities of the gas molecule and particle, respectively, in a fixed reference frame. Thus, if the particle shape dependence for $\sigma$ and the distribution function $f(\theta)$ can be found, then subject to the assumptions described above, the influence of particle shape on the rates of interphase momentum and energy transfer can be determined.

First consider the dependence of $f(\theta)$ on the particle shape. For an arbitrary convex particle, let the particle surface be divided into a large number $\mathrm{N}$ of flat surface elements, each of area $\partial \mathrm{A}$. Now assume that a gas molecule collides with the particle on a particular surface element $i$. If all orientations of the particle for which this collision may occur are equally likely, then the relative velocity vector of the incident molecule has an isotropic distribution over $\theta \in[0, \pi / 2]$. This vector will be contained within a solid angle element of size $\sin \theta d \theta d \phi$, where $\phi$ is the azimuthal angle relative to some reference direction in the plane of the surface 
element. Therefore $f(\theta)$ must be proportional to the size of the solid angle, so that $f(\theta) \propto \sin \theta$. Now, if we remove the requirement that the collision occurs on the surface element $i$, and only assume that a collision does occur somewhere on the particle surface, then the probability that the collision point will be located on element i must be proportional to the projected area of this element in the direction of the relative velocity vector $\mathbf{u}_{\mathrm{r}}$. Thus $f(\theta)$ is proportional to this projected area $\partial A \cos \theta$, so that $f(\theta) \propto \cos \theta$. By the above arguments, $f(\theta)$ must then be proportional to $\sin \theta \cos \theta$. Applying a trigonometric identity and the normalization condition $\int_{0}^{\pi / 2} f(\theta) d \theta=1$, we find that

$$
f(\theta)=\sin 2 \theta \text {. }
$$

As this distribution function is valid for any surface element, it must also be valid for the particle as a whole. Note that $f(\theta)$ will have no dependence on the particle shape, so long as the above assumptions are valid.

Next consider the particle shape dependence of the average collision cross section $\sigma$. As above, assume that the particle surface is made up of a large number $\mathrm{N}$ of flat surface elements, each with area $\partial \mathrm{A}$. Now define $\theta_{\mathrm{i}}$ as the angle between an outward normal vector on a given element $i$ and the relative velocity vector $\mathbf{u}_{\mathrm{r}}$ of an incident gas molecule. Under the assumption that the gas molecule is much smaller than the particle, the instantaneous collision cross section $\sigma$ ' will be the sum of the projected areas of all exposed faces:

$$
\sigma^{\prime}=\sum_{i=1}^{\mathrm{N}} \partial \mathrm{A} \max \left\{\cos \theta_{i}, 0\right\}
$$

The average collision cross section $\sigma$ can then be approximated as the average of a large number $\mathrm{M}$ of $\sigma$ ' values, each of which corresponds to a randomly chosen particle orientation relative to $\mathbf{u}_{\mathrm{r}}$. If $\theta_{\mathrm{ij}}$ is the value of $\theta$ on surface element $i$ for the jth realization of $\sigma^{\prime}$, then $\sigma$ can be given by

$$
\begin{aligned}
\sigma & =\lim _{\mathrm{M} \rightarrow \infty} \frac{1}{\mathrm{M}} \sum_{j=1}^{\mathrm{M}} \sum_{i=1}^{\mathrm{N}} \partial \mathrm{A} \max \left\{\cos \theta_{i j}, 0\right\} \\
& =\sum_{i=1}^{\mathrm{N}}\left(\partial \mathrm{A} \lim _{\mathrm{M} \rightarrow \infty} \frac{1}{\mathrm{M}} \sum_{j=1}^{\mathrm{M}} \max \left\{\cos \theta_{i j}, 0\right\}\right) \\
& =\sum_{i=1}^{\mathrm{N}}\left(\partial \mathrm{A} \int_{0}^{\pi} g\left(\theta_{i}\right) \max \left\{\cos \theta_{i}, 0\right\} d \theta_{i}\right)
\end{aligned}
$$

Here $g\left(\theta_{\mathrm{i}}\right)$ is the distribution function of $\theta_{\mathrm{i}}$ for a collision which may occur anywhere on the particle surface. From the solid angle argument used in the derivation of Eq. (3), it can be shown that $g\left(\theta_{\mathrm{i}}\right) \propto \sin \theta_{\mathrm{i}}$ if the particle has no preferred orientation relative to $\mathbf{u}_{\mathrm{r}}$. Applying the normalization condition $\int_{0}^{\pi} g\left(\theta_{\mathrm{i}}\right) d \theta_{\mathrm{i}}=1$, we find that $g\left(\theta_{\mathrm{i}}\right)=1 / 2 \sin \theta_{\mathrm{i}}$ for $\theta_{\mathrm{i}} \in[0, \pi]$, so $\int_{0}^{\pi} g\left(\theta_{i}\right) \max \left\{\cos \theta_{i}, 0\right\} d \theta_{i}=1 / 4$. If the total surface area of the particle is $\mathrm{A}_{\mathrm{s}}=\sum_{i=1}^{\mathrm{N}} \partial \mathrm{A}$ then substitution into Eq. (5) gives the final result that $\sigma=1 / 4 \mathrm{~A}_{\mathrm{s}}$. Thus, for a convex particle of arbitrary shape, the average collision cross section will be one fourth of the particle surface area.

As $f(\theta)$ has no particle shape dependence and $\sigma$ depends only on the particle surface area, then subject to the assumptions described above, any convex particle can be modeled as a spherical particle of the same surface area for calculations of interphase momentum and energy transfer. Following a standard convention ${ }^{17}$, let the particle shape be characterized by a shape factor $\psi \equiv \mathrm{A}_{\mathrm{o}} / \mathrm{A}_{\mathrm{s}}$, where $\mathrm{A}_{\mathrm{o}}$ is the surface area of a sphere with the same volume as the particle. Define $R_{o}$ as the radius of this same sphere, which for most particle shapes will be comparable to one-half of some characteristic average particle length. The effective particle radius $R_{p}$, for use in momentum and energy transfer calculations, can then be determined from known values of $\psi$ and $\mathrm{R}_{\mathrm{o}}$ through the following relation:

$$
R_{p}=R_{0} \psi^{-1 / 2}
$$

As $R_{p} \geq R_{0}$ it follows that a convex nonspherical particle in locally free molecular flow will behave like a spherical particle of equal mass but greater volume. Thus, the larger effective radius for a nonspherical particle will be accompanied by a reduction in the effective particle density. If the particle mass $M_{p}$ is calculated as $M_{p}=4 / 3 \pi \rho_{p} R_{p}{ }^{3}$ then the effective particle density $\rho_{p}$ can be found through the relation $\rho_{p}=\rho_{o} \psi^{3 / 2}$, where $\rho_{o}$ is the actual density of the particle material.

Through this analysis, the solid particle model of Gallis et al., as well as a two-way coupling method discussed below, can be extended and applied to a variety of nonspherical particles. While the analysis is not strictly valid for particles with highly complicated non-convex shapes, such as soot agglomerates, it is thought that this can provide at least a first-order approximation for the properties of such particles when included in a simulation.

\section{TWO-WAY COUPLING MODEL}

As discussed in the introduction, solid rocket plume flows and other two phase free expansion flows of interest are often characterized by a considerable transfer of momentum and energy between the gas and solid particles, such that the properties of each phase are significantly affected by the presence of the other. Under these conditions, the Gallis model assumption of one-way coupling is invalid, and the influence of particles on the surrounding gas must be considered. While the procedure outlined above may still be used to model the time-variation of particle properties, 
additional steps must be included in the calculations to account for potentially significant two-way coupling effects. The following analysis provides a physical model for the effect of an interphase collision on a gas molecule, and allows for a numerical procedure through which two-way coupling may be considered.

First, note that all assumptions listed above for the Gallis method are again used for consideration of momentum and energy coupling from a particle to the surrounding gas. Most importantly, the particle is assumed to be in a locally free molecular flow, so that any influence of reflected gas molecules on an incident molecule can be neglected during the collision process. The characteristics of the collision will therefore depend only on the properties of the particle and the single gas molecule involved in the collision. Further, all interphase collisions must involve either specular reflection or diffuse reflection with full thermal accommodation. While this is a relatively simplistic and phenomenological collision model, is has been shown experimentally to allow for a high degree of accuracy over a wide range of conditions, and is used as well in Eqs. (1) and (2).

Now consider the collision process between an individual gas molecule and a spherical solid particle. As shown above, the collision angle $\theta$ between the initial relative velocity vector $\mathbf{u}_{\mathrm{r}}$ and the local particle surface normal at the collision point will have a range of $[0, \pi / 2]$ and a distribution function given by Eq. (3). Let $\delta$ represent the deflection angle in the collision, defined as the angle between $-\mathbf{u}_{\mathrm{r}}$ and the post-collision relative velocity vector $\mathbf{u}_{\mathrm{r}}{ }^{*}=\mathbf{u}_{\mathrm{m}}{ }^{*}-\mathbf{u}_{\mathrm{p}}{ }^{*}$, where $\mathbf{u}_{\mathrm{m}}{ }^{*}$ and $\mathbf{u}_{\mathrm{p}}{ }^{*}$ are the absolute velocity vectors of the gas molecule and the particle respectively following the collision. (The superscript "*" is used here to denote any postcollision value.) Thus, a $\delta$ value of zero is equivalent to the relation $\mathbf{u}_{\mathrm{r}}{ }^{*}=-\mathbf{u}_{\mathrm{r}}$. For a collision involving specular reflection, any given $\theta$ will correspond to a $\delta$ value of

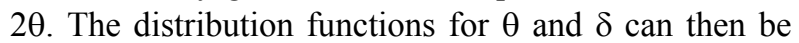
related by $f(\delta) \mathrm{d} \delta=f(\theta) \mathrm{d} \theta$, so that, from Eq. (3), the deflection angle for a specularly reflecting collision will have the following distribution:

$$
f(\delta)=1 / 2 \sin \delta \quad \text { for } \delta \in[0, \pi]
$$

Note that the azimuthal angle $\varepsilon$ of the vector $\mathbf{u}_{\mathrm{r}}{ }^{*}$, relative to a fixed direction in the plane normal to $\mathbf{u}_{\mathrm{r}}$, must have a uniform distribution over $[0,2 \pi]$. From comparison with the distribution function $g\left(\theta_{\mathrm{i}}\right)$ discussed above, it can therefore be shown that Eq. (7) corresponds to a total lack of directional dependence in $\mathbf{u}_{\mathrm{r}}{ }^{*}$. Thus, following a specularly reflecting collision, the relative velocity of the gas molecule will have a magnitude of $c_{r}=\left|\mathbf{u}_{\mathrm{r}}\right|$ and may be oriented with equal probability in any direction.

If the collision instead involves diffuse reflection, then the collision dynamics are far more complicated, and only a numerical approximation for the deflection angle distribution function $f(\delta)$ can be determined. In order to find this expression, two coordinate systems must now be used: First, let a coordinate system $(\mathrm{x}, \mathrm{y}, \mathrm{z})$ be defined so that the origin is at the particle center, the $y$-axis is parallel to the initial relative velocity vector $\mathbf{u}_{\mathrm{r}}$, and the collision point is located on the $x-y$ plane. For the second coordinate system $\left(x^{\prime}, y^{\prime}, z^{\prime}\right)$, the origin is at the collision point, the $y^{\prime}$-axis is along the local surface normal, and the particle center is on the $x^{\prime}$ '- $y$ ' plane. Both coordinate systems are shown in Fig (1), as are the relevant angles described below.

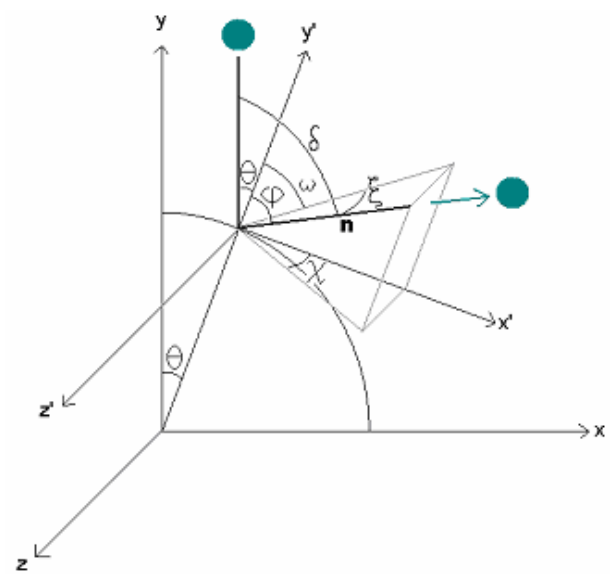

Figure 1. Coordinate systems and angles used in the evaluation of $f(\delta)$ for diffuse reflection.

Next, let $\varphi$ denote the angle between the postcollision relative velocity vector $\mathbf{u}_{\mathrm{r}}{ }^{*}$ and the $\mathrm{y}^{\text {'-axis, and }}$ designate as $\chi$ the azimuthal angle between the $\mathrm{x}^{\prime}$-axis and the projection of $\mathbf{u}_{\mathrm{r}}{ }^{*}$ onto the $\mathrm{x}^{\prime}-\mathrm{z}$ ' plane. While specular reflection requires that $\varphi=\theta$ and $\chi=0$, in the case of diffuse reflection $\varphi$ will have a continuous distribution over $[0, \pi / 2]$ and $\chi$ will be uniformly distributed over $[-\pi, \pi]$. Through further analysis, it can be shown that the probability that the post-collision trajectory of a diffusely reflecting molecule will be contained within the differential solid angle $\mathrm{d} \varphi \sin \varphi \mathrm{d} \chi$ centered at $(\varphi, \chi)$ must be proportional to both $\cos \varphi$ and the size of the solid angle. By imposing the normalization condition and a trigonometric identity, we find the following form for the distribution function of $\varphi$ :

$$
f(\varphi)=\sin (2 \varphi) \quad \text { for } \varphi \in[0, \pi / 2]
$$

As shown in the appendix, the angles $\theta, \varphi$, and $\chi$ can be related to the total deflection angle $\delta$ by:

$$
\cos \delta=(\cos \theta-\sin \theta \tan \varphi \cos \chi)\left[\frac{1-(\sin \varphi \sin \chi)^{2}}{1+(\tan \varphi \cos \chi)^{2}}\right]^{1 / 2}
$$


A Monte Carlo integration method may be employed to determine the shape of the distribution function for $\delta$. Values of $\theta$ and $\phi$ are generated by applying the acceptance-rejection method ${ }^{9}$ to Eqs. (3) and (8), and $\chi$ values are randomly generated with uniform probability over the range $[-\pi, \pi]$. Eq. (9) is then used to calculate the corresponding values of $\delta$. These values are sorted into bins of finite width $\Delta \delta$, and the frequency that $\delta$ values fall within each bin is recorded to produce a histogram that approximates the shape of the distribution function for $\delta$ over $[0, \pi]$. The resulting shape is found to be closely approximated by the following sixth-order polynomial:

$$
\begin{aligned}
f(\delta)= & 0.02042 \delta^{6}-0.2515 \delta^{5}+1.104 \delta^{4} \\
& -1.903 \delta^{3}+0.4938 \delta^{2}+1.248 \delta
\end{aligned}
$$

As the deflection angle $\delta$ and post-collision relative speed $\mathrm{c}_{\mathrm{r}}{ }^{*}$ can be shown to be statistically independent in a diffusely reflecting collision, Eq. (10) is valid for any molecule-particle collision pair for which diffuse reflection is involved. Note that a spherical particle has been used here for simplicity, but the above analysis allows Eq. (10) to be extended to a range of nonspherical particles. Both the numerical solution and the polynomial approximation are shown in Fig. (2), along with the equivalent distribution function for specular reflection.

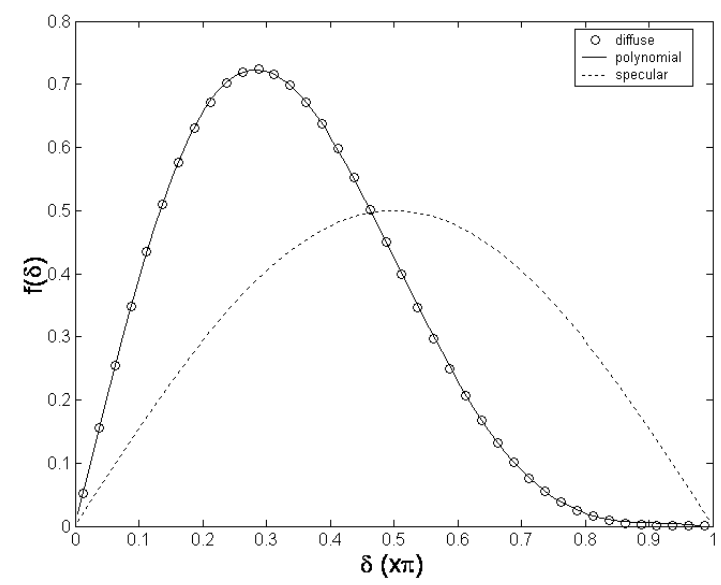

Figure 2. Comparison of distribution functions for deflection angle $\delta$.

The above distribution functions are utilized in the following procedure, which allows a solid particle in a two phase DSMC simulation to influence the surrounding gas. We first determine which, if any, computational gas molecules will collide with the particle during each time step. A modification of the No Time Counter method of $\operatorname{Bird}^{9}$ is used to find the number $\mathrm{n}_{\mathrm{s}}$ of computational gas molecules that are selected as potential collision partners for the particle. The value of $n_{s}$ is roughly given by

$$
\mathrm{n}_{\mathrm{s}} \approx \mathrm{N}_{\mathrm{p}} \mathrm{n}_{\mathrm{g}} \pi \mathrm{R}_{\mathrm{p}}^{2}\left(\mathrm{c}_{\mathrm{r}}\right)_{\max } \Delta \mathrm{t} / \mathrm{V}_{\mathrm{c}}
$$

where $\mathrm{N}_{\mathrm{p}}$ is the number of actual solid particles represented by the computation particle, $\mathrm{n}_{\mathrm{g}}$ is the number of computational gas molecules assigned to the same grid cell as the particle, $R_{p}$ is the effective particle radius, $\Delta \mathrm{t}$ is the time step, $\mathrm{V}_{\mathrm{c}}$ is the cell volume, and $\left(c_{r}\right)_{\max }$ is the maximum pre-collision relative speed, over a large number of time steps, for any molecule-particle pair in this cell. Note that $\mathrm{n}_{\mathrm{s}}$ must be an integer, so a probabilistic sampling method is used to round the right side of (11) either up or down such that the average values of both sides are equal. Once $n_{s}$ molecules have been chosen as potential collision partners, those that do collide are selected with probability $\mathrm{c}_{\mathrm{r}} /\left(\mathrm{c}_{\mathrm{r}}\right)_{\max }$. It can be shown that this selection scheme corresponds to a probability $\mathrm{P}_{\text {coll }}=\pi \mathrm{N}_{\mathrm{p}} \mathrm{R}_{\mathrm{p}}{ }^{2} \mathrm{c}_{\mathrm{r}} \Delta \mathrm{t} / \mathrm{V}_{\mathrm{c}}$ that the particle will collide with a given molecule in the cell. Due to time step limitations inherent in DSMC, it has been found that $\mathrm{P}_{\text {coll }}$ values are almost universally several orders of magnitude smaller than one, so that the number of collisions per particle per time step is usually zero and is rarely greater than one.

If a given computational gas molecule is found to collide with the particle, then the collision is determined to involve either isothermal diffuse reflection, with a probability equal to the particle thermal accommodation coefficient $\tau$, or specular reflection, with probability $1-\tau$. If a specularly reflecting collision takes place, then the relative speed $\mathrm{c}_{\mathrm{r}}$ is unchanged in the collision, and the post-collision relative velocity $\mathbf{u}_{\mathrm{r}}{ }^{*}$ is found by multiplying $\mathrm{c}_{\mathrm{r}}$ by a unit vector $\mathbf{n}$ which is sampled from an isotropic distribution. (An efficient algorithm for calculating $\mathbf{n}$ is described in Ref. (9).) If diffuse reflection occurs, then the acceptance-rejection method is applied to Eq. (10) to find a value for $\delta$, and the azimuthal angle $\varepsilon$ of the post-collision relative velocity $\mathbf{u}_{\mathrm{r}}{ }^{*}$ around the initial relative velocity vector $\mathbf{u}_{\mathrm{r}}$ is randomly generated from a uniform distribution over $[0,2 \pi]$. As kinetic energy is not conserved in diffusely reflecting collisions, the post-collision relative speed $\mathrm{c}_{\mathrm{r}}{ }^{*}$ cannot be assumed to equal the initial relative speed $c_{r}$. Instead, a value of $c_{r}{ }^{*}$ must be determined by applying the acceptancerejection method to the distribution function

$$
f\left(c_{\mathrm{r}}^{*}\right)=2 \beta^{4} \mathrm{c}_{\mathrm{r}}^{* 3} \exp \left(-\beta^{2} \mathrm{c}_{\mathrm{r}}{ }^{* 2}\right)
$$

where $\beta=\left[\mathrm{m} /\left(2 \mathrm{k}_{\mathrm{B}} \mathrm{T}_{\mathrm{p}}\right)\right]^{1 / 2}$ is the inverse of the gas thermal speed scale at the particle temperature. For the case of a diffusely reflected polyatomic gas molecule, the post-collision value of the rotational energy $e_{\text {rot }}$ must also be altered. From Eq. (C16) in Ref. (9), the rotational energy of a diffusely reflected diatomic molecule can be calculated as

$$
\mathrm{e}_{\mathrm{rot}}=-\ln \left(\mathrm{R}_{\mathrm{f}}\right) \mathrm{k}_{\mathrm{B}} \mathrm{T}_{\mathrm{P}}
$$

where $R_{f}$ is a randomly generated number in $(0,1]$. As noted above, vibrational activation is assumed to be 
negligible for all flows of interest, so that no vibrational terms are included in Eq. (2) and conservation of energy requires that the gas molecule vibrational energy not be altered during an interphase collision.

Now let $u_{r}, v_{r}$, and $w_{r}$ be the components of $\mathbf{u}_{r}$ in the global coordinate system used in the simulation. The corresponding components of $\mathbf{u}_{\mathrm{r}}{ }^{*}$ can be computed from the values of $\mathrm{u}_{\mathrm{r}}, \mathrm{v}_{\mathrm{r}}, \mathrm{w}_{\mathrm{r}}, \mathrm{c}_{\mathrm{r}}$ and $\mathrm{c}_{\mathrm{r}}{ }^{*}$, and the angles $\delta$ and $\varepsilon$ using modifications of equations derived by Bird ${ }^{9}$ for binary elastic collisions. As modified for use with the above variables, these equations are written as:

$$
\begin{aligned}
& \mathrm{u}_{\mathrm{r}}^{*}=\frac{\mathrm{c}_{\mathrm{r}}^{*}}{\mathrm{c}_{\mathrm{r}}}\left[-\mathrm{u}_{\mathrm{r}} \cos \delta+\sin \delta \sin \varepsilon\left(\mathrm{v}_{\mathrm{r}}^{2}+\mathrm{w}_{\mathrm{r}}^{2}\right)^{1 / 2}\right] \\
& \mathrm{v}_{\mathrm{r}}^{*}=\frac{\mathrm{c}_{\mathrm{r}}^{*}}{\mathrm{c}_{\mathrm{r}}}\left[\frac{-\mathrm{v}_{\mathrm{r}} \cos \delta+\sin \delta\left(\mathrm{c}_{\mathrm{r}} \mathrm{w}_{\mathrm{r}} \cos \varepsilon-\mathrm{u}_{\mathrm{r}} \mathrm{v}_{\mathrm{r}} \sin \varepsilon\right)}{\left(\mathrm{v}_{\mathrm{r}}^{2}+\mathrm{w}_{\mathrm{r}}^{2}\right)^{1 / 2}}\right] \\
& \mathrm{w}_{\mathrm{r}}^{*}=\frac{\mathrm{c}_{\mathrm{r}}^{*}}{\mathrm{c}_{\mathrm{r}}}\left[\frac{-\mathrm{w}_{\mathrm{r}} \cos \delta-\sin \delta\left(\mathrm{c}_{\mathrm{r}} \mathrm{v}_{\mathrm{r}} \cos \varepsilon+\mathrm{u}_{\mathrm{r}} \mathrm{w}_{\mathrm{r}} \sin \varepsilon\right)}{\left(\mathrm{v}_{\mathrm{r}}^{2}+\mathrm{w}_{\mathrm{r}}^{2}\right)^{1 / 2}}\right]
\end{aligned}
$$

Eqs. (14) are evaluated to find $\mathbf{u}_{\mathrm{r}}{ }^{*}$ if the collision involves diffuse reflection. Once the components of $\mathbf{u}_{\mathrm{r}}{ }^{*}$ have been calculated for either type of collision, the absolute gas molecule velocity is updated to a final value of $\mathbf{u}_{\mathrm{m}}{ }^{*}=\mathbf{u}_{\mathrm{r}}{ }^{*}+\mathbf{u}_{\mathrm{p}}$, where $\mathbf{u}_{\mathrm{p}}$ is the velocity assigned to the solid particle. Note that the collision-induced velocity difference for the particle is assumed much smaller than that of the molecule. This follows from the previous assumption that the particle is much more massive than the particle, and allows the true postcollision particle velocity $\mathbf{u}_{\mathrm{p}}{ }^{*}$ to be replaced by $\mathbf{u}_{\mathrm{p}}$ for the calculation of $\mathbf{u}_{\mathrm{m}}{ }^{*}$.

This procedure is repeated for each solid particle during every time step. It can be shown, by integration of the distribution functions given above, that the average momentum and energy imparted on a gas molecule through a collision are equal in magnitude, respectively, to the average momentum and energy transfer rates to the solid particle (given by Eqs. (1) and (2)) multiplied by the ratio of the time step $\Delta$ t to the collision probability $\mathrm{P}_{\text {coll }}$. This property has been verified for both specular and diffuse reflection, and confirms that the method described here is consistent with the force and heat transfer equations of Gallis et al. In addition, and in contrast to the one-way coupled method described above, the total momentum and energy of the flow are now both conserved in an average sense.

\section{EXAMPLE SIMULATION AND RESULTS}

In order to demonstrate the consistency of this new method with the one-way coupling method discussed above, a sample simulation is performed. All flow properties are based on those expected along the axis and just beyond the nozzle exit plane in the exhaust flow of a small solid propellant rocket. The simulation geometry is simplified in order to isolate the effects of gas-particle interaction, and only a small domain is considered to limit the computational expense. The simulation is performed on a rectangular twodimensional grid, consisting of $0.1 \mathrm{~mm}$ long uniform inflow and outflow boundaries, separated on either end by $20 \mathrm{~mm}$ long specularly reflecting walls. The grid geometry is shown in Fig. (3). As no energy or longitudinal momentum may be transferred through the walls, it can be expected that, if the new two-way coupling method is physically consistent, the total momentum and energy flux will be the same over any transverse plane which passes through the grid.

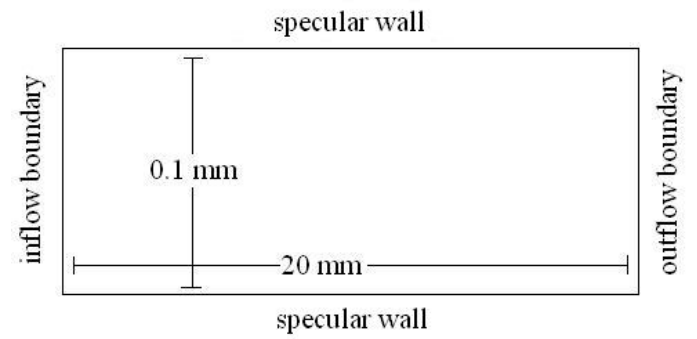

Figure 3. Grid dimensions and boundary types.

The gas in this simulation is a mixture of $\mathrm{H}_{2}$, $\mathrm{CO}$ and $\mathrm{N}_{2}$, with inflow number densities of $2 \times 10^{23} \mathrm{~m}^{-3}$, $1 \times 10^{23} \mathrm{~m}^{-3}$ and $1 \times 10^{23} \mathrm{~m}^{-3}$ respectively. At the inflow boundary the gas is assigned a bulk speed of $2000 \mathrm{~m} / \mathrm{s}$ and a temperature of $1000 \mathrm{~K}$. The solid phase consists of spherical alumina particles, of diameter $3 \times 10^{-6} \mathrm{~m}$ and $6 \times 10^{-6} \mathrm{~m}$, with a mass flow fraction of $40 \%$ divided equally between particles of either size. All particles have a velocity of $1200 \mathrm{~m} / \mathrm{s}$ and a temperature of 2200 $\mathrm{K}$ at the inflow boundary, with a total particle mass flow rate of $13.33 \mathrm{~kg} / \mathrm{s}-\mathrm{m}^{2}$. The particle material density is set as $3970 \mathrm{~kg} / \mathrm{m}^{3}$, with a specific heat of 765 $\mathrm{J} / \mathrm{kg}-\mathrm{K}$ and a surface thermal accommodation coefficient of 0.89 . The grid is divided into 5000 square cells of length $2 \times 10^{-5} \mathrm{~m}$, or approximately two mean free paths. Collisions within the gas phase are considered using the Variable Hard Sphere model, with reference molecular diameters given by Bird ${ }^{9}$. The time step size is $1.5 \times 10^{-9} \mathrm{~s}$ and the relative weights $\mathrm{N}_{\mathrm{g}}$ and $\mathrm{N}_{\mathrm{p}}$ are set so that, at steady state, about 270,000 computational gas molecules and 10,000 solid particles are contained within the grid. This corresponds to roughly 54 computational gas molecules and 2 solid particles per cell. Once steady state has been reached, various gas and particle properties are evaluated, and averaging is performed over approximately 200,000 time steps.

Simulation results are shown in Figs. (4), (5) and (6), based on values extracted along a line between the centers of the inflow and outflow boundaries. Due to the relatively small changes in the characteristics of either phase expected over the limited grid domain, the

American Institute of Aeronautics and Astronautics Approved for public release; distribution unlimited 
interphase transfer of momentum and energy should be nearly constant with downstream distance, so that most flow properties will vary linearly through the grid. The expected linear variation is observed in Fig. (4), in which the average gas and particle speeds are plotted against longitudinal distance from the inflow boundary. The slower particle phase is found to accelerate at a nearly constant rate, while the faster gas decelerates as momentum is transferred to the particles. Fig. (5) shows the corresponding trends in the gas and particle number densities. Neglecting the significant statistical scatter, the particle number density is found to decrease approximately linearly and the gas number density is found to increase linearly, as is expected from comparison with Fig. (4) and considerations of mass conservation. In Fig. (6) the longitudinal variations in the average particle temperature and gas translational temperature are shown. The gas and particle temperatures are found to increase and decrease respectively with downstream distance, as energy is transferred to the gas from the higher temperature particles.

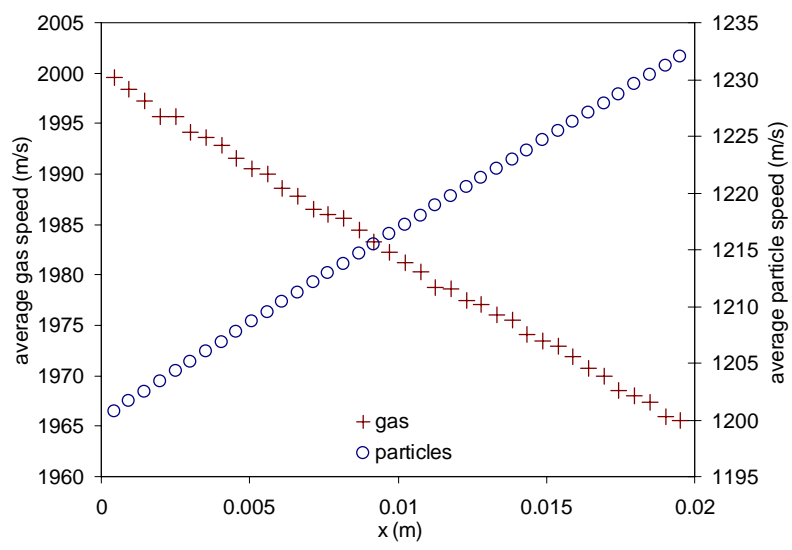

Figure 4. Longitudinal variation in average gas and particle speeds.

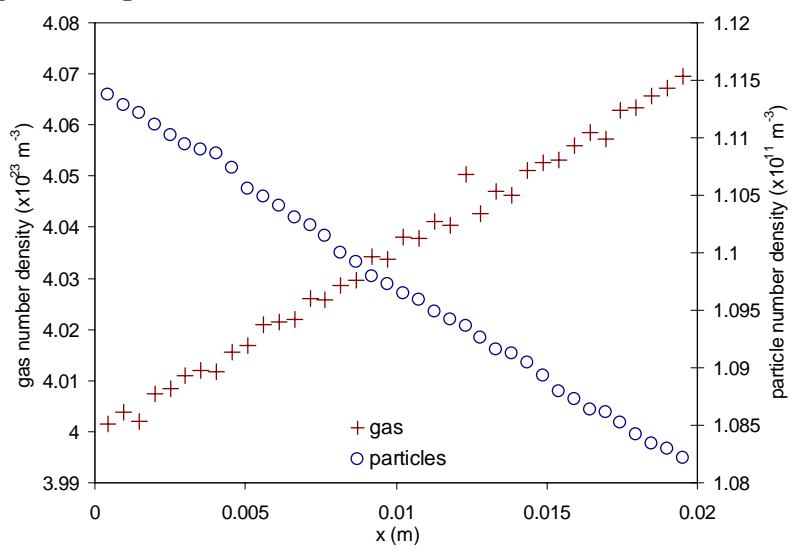

Figure 5. Gas and particle number densities.

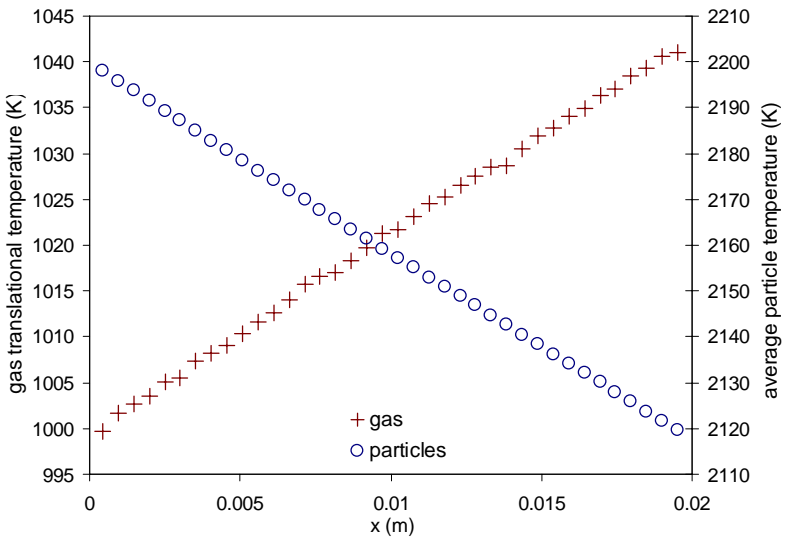

Figure 6. Variation in gas and particle temperatures.

As noted above, the accuracy and consistency of the two-way coupling method can be determined by verifying that momentum and energy are transferred between the two phases at equal rates, so that the total momentum or energy transfer rate will be the same through any transverse plane which intersects the grid domain. In order to show this, momentum and energy transfer rates of each phase are calculated along nine equally spaced planes. While approximations for these rates could be easily found through algebraic manipulations of the cell-averaged velocities, densities, and temperatures, the small size of the grid and the extreme sensitivity of flux values to statistical scatter require that a more direct approach be used. The alternate procedure is as follows: During every time step for which sampling is performed, it is determined which computational gas molecules or solid particles pass through each of the nine planes. Values are recorded for the mass, momentum, and energy transferred through each plane, to which the mass, longitudinal momentum, kinetic energy, and internal energy of each of these objects is either added or subtracted, depending on the direction in which the object passes through the plane. Resulting values are then divided by the time step size $\Delta \mathrm{t}$, and averaging is performed over all sampling time steps. Each timeaveraged momentum and energy transfer value is then divided by the ratio of the corresponding mass transfer rate to the average mass transfer rate assigned at the inflow boundary (equal to $0.002 \mathrm{~kg} / \mathrm{s}$ for the gas and $0.001333 \mathrm{~kg} / \mathrm{s}$ for the particles).

Note that this last step is required to account for statistical fluctuations in the number of objects which pass through these planes. This is also necessary to correct for a slight reduction in the gas number flux with downstream distance, due to the fact that computational gas molecules may exit the grid through the inflow boundary. The correction is only on the order of $0.1 \%$, but is found to significantly improve the 
accuracy of results, as the values of interest will vary only slightly through the length of the grid.

The variation in longitudinal momentum transfer rates is shown in Fig. (7). While different scales are used for the particles and gas, the ranges of both scales are equal, so that trends in the two profiles can be easily compared. As expected, momentum is observed to be removed from the gas at nearly the exact rate that momentum is added to the particle phase. Both data sets are closely approximated by linear leastsquares trend lines, with slopes that differ in magnitude by less than $2 \%$. Similar trends can be found in Fig. (8), which shows the variation in energy transfer rates with downstream distance. Again, the magnitudes of linear trend lines are nearly equal, and energy is observed to be removed from the particles at approximately the same rate that energy is added to the gas.

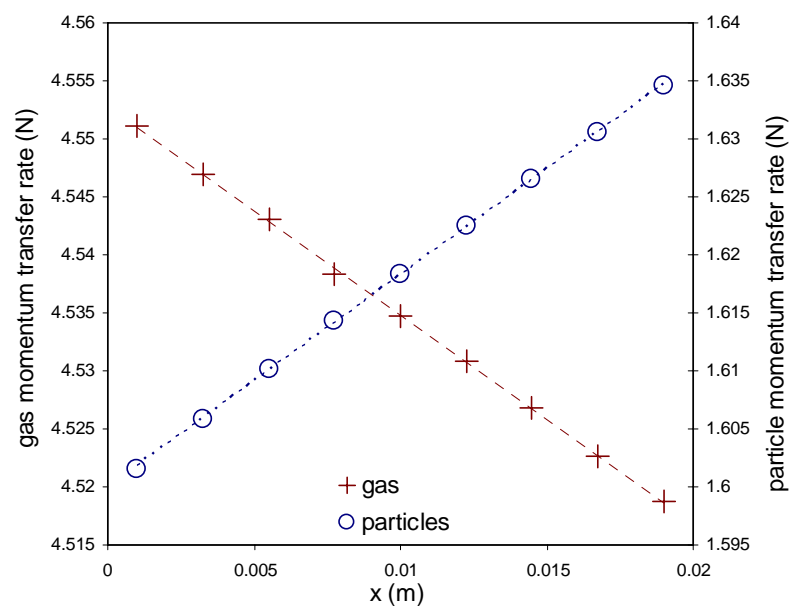

Figure 7. Variation in longitudinal momentum transfer rates with downstream distance.

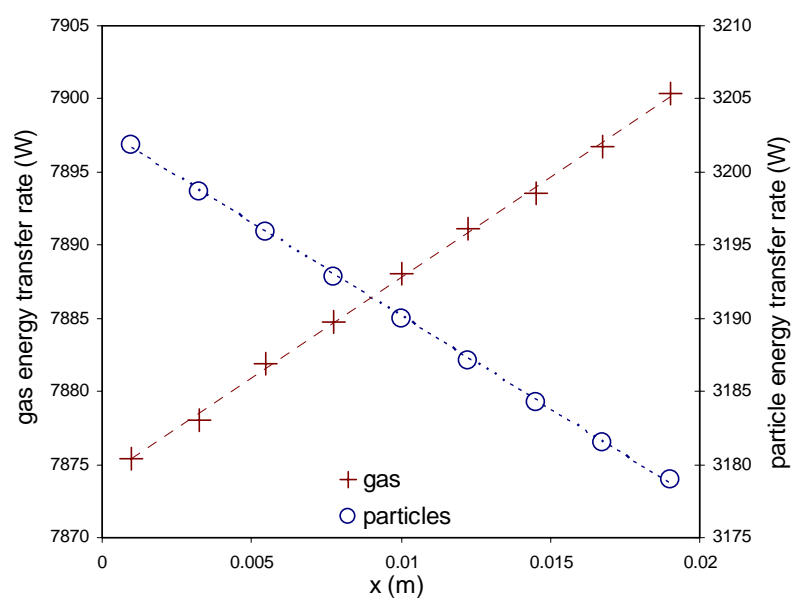

Figure 8. Energy transfer rates for gas and particles.

The small discrepancies which are observed in Figs. (7) and (8), and the local variation in the total momentum and energy transfer rates which these discrepancies imply, can be explained by a number of factors. First, some error may be due to the collision selection scheme given by Eq. (11), as gas molecules most likely to collide with a given particle may not be chosen as potential collision partners. This may slightly reduce the interphase collision frequency, and is a problem inherent in the No Time Counter method on which the collision selection scheme is based. A more likely error source is the fact that momentum and energy are conserved only in a time-averaged sense, as the instantaneous momentum and energy transfer arising from a single interphase collision is not modeled in the same manner for calculations used to alter properties of the two different phases. This difference in collision modeling arises from the wide disparity expected in number density between solid particles and gas molecules, so that the interphase collision frequency for a single solid particle is likely several orders of magnitude larger than that of a gas molecule. (For the simulation described here, these two collision frequencies differ by a factor of about $4 \times 10^{12}$.)

The lack of exact momentum and energy conservation should give rise to random walk errors, which are magnified in the results of Figs. (7) and (8) due to the extreme numerical sensitivity of the observed trends. However, these errors are shown to be relatively small, and are expected to further decrease when the sampling period is lengthened. As the DSMC method generally requires time (or ensemble) averaging over a large number of time steps, instantaneous momentum and energy conservation should not be required to achieve levels of accuracy in the simulation results within the expected statistical scatter. It can therefore be assumed that Figs. (7) and (8) do adequately demonstrate the conservation of momentum and energy, so that the two-way coupling method is physical consistent. The initial one-way coupling method is shown in Refs. (12) and (14) to exhibit a high degree of accuracy, so it follows from the above arguments that the new method should be reasonably accurate as well.

\section{CONCLUSIONS AND FUTURE WORK}

An outline has been provided for the implementation of the method of Gallis et al. to simulate one-way coupled two phase rarefied flows, and the method has been extended for use with a range of nonspherical particles. A new method has been developed in order to account for two-way coupling effects, and to broaden the range of flow regimes which may be accurately modeled. It is thought that the method described here is the first to allow for the simulation of two-way coupled two phase flows involving a highly nonequilibrium gas. These conditions are commonly found in high altitude plume flows from solid propellant rockets, and may also occur in spacecraft fuel venting and thruster flows. The 
method therefore holds promise as an important tool in the analysis of a variety of multiphase high altitude plumes.

The work discussed here is part of an ongoing project to develop and implement accurate modeling techniques for high altitude plume and fuel venting flows. Future studies will consider reductions in computational cost through a series of interphase coupling parameters, as well as the implementation of models for particle formation, surface chemistry, and phase change. A detailed radiation model will be developed in order to accurately account for radiative heat transfer from and within the particle phase, and to provide capabilities for the analysis of plume radiation signatures. These models will be used in a variety of large scale simulations, which will also be developed in future work.

\section{ACKNOWLEDGMENTS}

The authors gratefully acknowledge the financial support for this work provided by the Air Force Research Laboratory at Edwards Air Force Base, with Dean Wadsworth and Tom Smith as technical monitors.

\section{REFERENCES}

1. Simmons, F. S., Rocket Exhaust Plume Phenomenology, Aerospace Press, El Segundo, CA, 2000, pp. 173-200.

2. Rattenni, L., "Solid Motor Plume Analyses for the STAR-1 Spacecraft," AIAA Paper 20003190, 2000.

3. Ciucci, A., and Iaccarino, G., "Numerical Analysis of the Turbulent Flow and Alumina Particle Trajectories in Solid Rocket Motors," AIAA Paper 97-2860, 1997.

4. Beiting, E., "Predicted Physical and Optical Characteristics of Solid Rocket Motor Exhaust in the Stratosphere," AIAA Paper 97-0532, 1997.

5. Kovalev, O., "Motor and Plume Particle Size Prediction in Solid-Propellant Rocket Motors," Journal of Propulsion and Power, Vol. 18, No. 6, 2002, pp.1199-1210.

6. Rodionov, A. V., Plastinin, Y. A., Drakes, J. A., Simmons, M. A., and Hiers, R. S., "Modeling of Multiphase Alumina-Loaded Jet Flow Fields," AIAA Paper 98-3462, 1998.

7. York, B. J., Lee, R. A., Sinha, N., and Dash, S. M., "Progress in the Simulation of Particulate Interactions in Solid Propellant Rocket Exhausts," AIAA Paper 2001-3590, 2001.

8. Dash, S. M., Wolf, D. E., Beddini, R. A., and Pergament, H. S., "Analysis of Two-Phase Flow Processes in Rocket Exhaust Plumes,"
Journal of Spacecraft and Rockets, Vol. 22, No. 3, 1985, pp. 367-380.

9. Bird, G. A., Molecular Gas Dynamics and the Direct Simulation of Gas Flows, Clarendon Press, Oxford, 1994, pp. 36, 203-204, 218220, 408-409, 425, 428.

10. Boyd, I. D., Penko, P. F., Meissner, D. L., and DeWitt, K. J., "Experimental and Numerical Investigations of Low-Density Nozzle and Plume Flows of Nitrogen," AIAA Journal, Vol. 30, No. 10, 1992, pp. 2453-2461.

11. Penko, P. F., Boyd, I. D., Meissner, D. L., and DeWitt, K. J., "Measurement and Analysis of a Small Nozzle Plume in Vacuum," Journal of Propulsion and Power, Vol. 9, No. 4, 1993, pp. 646-648.

12. Gallis, M. A., Torczynski, J. R., and Rader, D. J., "An Approach for Simulating the Transport of Spherical Particles in a Rarefied Gas Flow via the Direct Simulation Monte Carlo Method," Physics of Fluids, Vol. 13, No. 11, 2001, pp. 3482-3492.

13. Dietrich, S., and Boyd, I. D., "Scalar and Parallel Optimized Implementation of the Direct Simulation Monte Carlo Method," Journal of Computational Physics, Vol. 126, 1996, pp. 328-342.

14. Burt, J. M., and Boyd, I. D., "Evaluation of a Monte Carlo Model for Two Phase Rarefied Flows," AIAA Paper 2003-3496, 2003.

15. Israel, G., and Friedlander, K., "High-Speed Beams of Small Particles," Journal of Colloid and Interface Science, Vol. 24, 1967, pp. 330337.

16. Kassal, T. T., "Scattering Properties of Ice Particles Formed by Release of $\mathrm{H}_{2} \mathrm{O}$ in Vacuum," Journal of Spacecraft, Vol.11, No. 1, 1974, pp. 54-56.

17. Crowe, C., Sommerfeld, M., and Tsuji, Y., Multiphase Flows with Droplets and Particles, CRC Press, New York, 1998, pp. 92.

\section{APPENDIX}

A proof is provided for the relation between the angles $\theta, \phi, \chi$, and $\delta$ given as Eq. (9), based on the geometry shown in Fig. (1). First, define a unit vector $\mathbf{n}$ in the direction of the post-collision relative velocity vector $\mathbf{u}_{\mathrm{r}}{ }^{*}$, with components $\left(\mathrm{n}_{\mathrm{x}}, \mathrm{n}_{\mathrm{y}}, \mathrm{n}_{\mathrm{z}}\right)$ and $\left(\mathrm{n}_{\mathrm{x}}, \mathrm{n}_{\mathrm{y}^{\prime}}, \mathrm{n}_{\mathrm{z}}\right)$ in the $(\mathrm{x}, \mathrm{y}, \mathrm{z})$ and $\left(\mathrm{x}^{\prime}, \mathrm{y}^{\prime}, \mathrm{z}^{\prime}\right)$ coordinate systems respectively. Two additional angles must also be defined: Denote as $\xi$ the angle between $\mathbf{u}_{\mathrm{r}}{ }^{*}$ and the projection of $\mathbf{u}_{\mathrm{r}}{ }^{*}$ onto the $\mathrm{x}^{\prime}-\mathrm{y}^{\prime}$ plane, and let $\omega$ be the angle between this projection and the $y^{\prime}$-axis.

The following expressions can be found for $\omega$, $\chi$, and $\varphi$ in terms of the components of $\mathbf{n}$ : 
$\tan \omega=\mathrm{n}_{\mathrm{x}^{\prime}} / \mathrm{n}_{\mathrm{y}^{\prime}} \quad \tan \varphi=\sqrt{\mathrm{n}_{\mathrm{x}^{\prime}}^{2}+\mathrm{n}_{\mathrm{z}^{\prime}}^{2}} / \mathrm{n}_{\mathrm{y}^{\prime}} \quad \cos \chi=\mathrm{n}_{\mathrm{x}^{\prime}} / \sqrt{\mathrm{n}_{\mathrm{x}^{\prime}}^{2}+\mathrm{n}_{\mathrm{z}^{\prime}}^{2}}$

Substitution then gives

$\tan \omega=\tan \varphi \cos \chi$

Similarly, the relations

$\sin \xi=\mathrm{n}_{\mathrm{z}^{\prime}} \quad \sin \varphi=\sqrt{\mathrm{n}_{\mathrm{x}^{\prime}}^{2}+\mathrm{n}_{\mathrm{z}^{\prime}}^{2}} \quad \sin \chi=\mathrm{n}_{\mathrm{z}^{\prime}} / \sqrt{\mathrm{n}_{\mathrm{x}^{\prime}}^{2}+\mathrm{n}_{\mathrm{z}^{\prime}}^{2}}$

are used to find an expression for $\xi$ in terms of $\varphi$ and $\chi$ :

$\sin \xi=\sin \varphi \sin \chi$

Next, $\delta$ can be related to $\theta, \omega$, and $\xi$ by recognizing that

$\cos \delta=\mathrm{n}_{\mathrm{y}} \quad \cos (\theta+\omega)=\mathrm{n}_{\mathrm{y}} / \sqrt{\mathrm{n}_{\mathrm{x}}^{2}+\mathrm{n}_{\mathrm{y}}^{2}} \quad \cos \xi=\sqrt{\mathrm{n}_{\mathrm{x}}^{2}+\mathrm{n}_{\mathrm{y}}^{2}}$

Then by substitution and a trigonometry identity:

$$
\begin{aligned}
\cos \delta & =\cos \xi \cos (\theta+\omega) \\
& =\cos \xi(\cos \theta \cos \omega-\sin \theta \sin \omega)
\end{aligned}
$$

Note that both $\xi$ and $\omega$ are confined to the range $[-\pi / 2$, $\pi / 2]$, hence $\cos \xi=\sqrt{1-\sin ^{2} \xi}, \cos \omega=\left(1+\tan ^{2} \omega\right)^{-1 / 2}$, and $\sin \omega=\tan \omega\left(1+\tan ^{2} \omega\right)^{-1 / 2}$. Using these relations, Eqs. (15) and (16) are substituted into Eq. (17) to give an expression for $\delta$ in terms of $\theta, \chi$, and $\varphi$. After some algebraic simplification, this expression can be written as

$$
\cos \delta=(\cos \theta-\sin \theta \tan \varphi \cos \chi)\left[\frac{1-(\sin \varphi \sin \chi)^{2}}{1+(\tan \varphi \cos \chi)^{2}}\right]^{1 / 2} .
$$

